\title{
Laser Desorption/Ionization Mass Spectrometry on Porous Silica and Alumina for Peptide Mass Fingerprinting
}

\author{
Nawar Shenar, Jean Martinez, and Christine Enjalbal \\ Institut des Biomolécules Max Mousseron (IBMM), UMR 5247 CNRS-Universités Montpellier 1 et 2, \\ Université Montpellier 2, Montpellier, France
}

\begin{abstract}
We investigated a variant of desorption/ionization on porous silicon (DIOS) mass spectrometry utilizing an aqueous suspension of either porous silica gel or porous alumina (pore size of 60 and $90 \AA$, respectively). Laser desorption/ionization (LDI) from samples directly deposited on a stainless steel surface without any inorganic substrates was also achieved. Synthetic peptides designed to cover large sequence diversity constituted our model compounds. Sample preparation, including material conditioning, peptide solubilization, and deposition protocol onto standard matrix-assisted laser desorption/ionization (MALDI) probe, as well as ionization source tuning were optimized to perform sensitive reproducible LDI analyses. The addition of either a cationizing agent or an alkali metal scavenger to the sample suspension allowed modification of the ionization output. Comparing hydrophilic silica gel to hydrophobic reversed-phase silica gel as well as increasing material pore size provided further insights into desorption/ionization processes. Furthermore, mixtures of peptides were analyzed to probe the spectral suppression phenomenon when no interfering organic matrix was present. The results gathered from synthetic peptide cocktails indicated that LDI mass spectrometry on silica gel or alumina constitutes a promising complementary method to MALDI in proteomics for peptide mass fingerprinting. (J Am Soc Mass Spectrom 2008, 19, 632-644) (C) 2008 American Society for Mass Spectrometry
\end{abstract}

$\mathrm{A}$ laser desorption/ionization (LDI) approach [1-9] was investigated by Siuzdak and collaborators using porous silicon as the substrate [10]. This desorption/ionization on porous silicon (DIOS) mass spectrometry [10] allows the analysis of various compounds, such as small organic molecules [11], amino acids [12], peptides [13], and fatty acids [14]. More recently, other research groups achieved direct desorption/ionization from carbon nanotubes [15], fullerenes [16], Au clusters [17], silicon nanopowder [18], and porous alumina [19]. These LDI methods, sometimes referred to as "matrix-free", produce mass spectra that exhibit less matrix ions in the low mass range compared with the chemical noise generated by the organic matrices used in matrix-assisted laser desorption/ionization (MALDI) mass spectrometry. Such chemical noise that usually pollutes the low mass range of MALDI mass spectra and that may complicate the detection of small molecules is less pronounced in the DIOS mass spectra.

Nevertheless, the drawback of such an approach is in the preparation of the silicon material. To present

Address reprint requests to Professor C. Enjalbal, Institut des Biomolécules Max Mousseron (IBMM), UMR 5247 CNRS-Universités Montpellier 1 et 2, Université Montpellier 2, Place Eugène Bataillon, 34095 Montpellier Cedex 5, France. E-mail: enjalbal@univ-montp2.fr adequate physical properties [20], silicon surfaces are conditioned via a galvanostatic etching procedure from Si wafer to produce pores with nanometer diameters. Commercially available ready-to-use DIOS-chips can also be mounted on modified MALDI plates [20]. Otherwise, silicon powders ( 5 to $50 \mathrm{~nm}$ particle size) were investigated as a substitute to DIOS chips to make the overall sample preparation simpler and safer [18]. Provided that the appropriate powder preparation was performed (including particle etching by $\mathrm{HF}$, oxidation by $\mathrm{HNO}_{3}$, and derivatization of the generated $\mathrm{SiOH}$ groups), such silicon nanopowder method was found to exhibit the same characteristics as DIOS mass spectrometry.

Porous silicon is a material that presents (1) a large active surface and (2) a strong absorbance in the UV range. Such a form of silicon was thus found particularly suitable for LDI mass spectrometry with irradiation at $337 \mathrm{~nm}$, the pore size and the surface chemical nature being the most important parameters as stated by Siuzdak and coworkers in their early publications on DIOS [20]. The influence on DIOS performance of the porous silicon surface oxidation that changes the surface chemical characteristics was also questioned [11]. Standard DIOS chips that display hydrophobic $\mathrm{SiH}$ moieties on the surface, and hydrophilic porous silicon dioxide chips were compared [12, 13]. Whereas similar 
results in terms of signal intensity and resolution were initially reported from oxidized silicon surfaces [13], different responses according to the compound hydrophobicity were thereafter obtained by other research groups for small molecule analysis [12]. Based on these results, it seems that silicon dioxide $\left(\mathrm{SiO}_{2}\right)$, also named silica, constitutes an interesting alternative to porous silicon and silicon powder studied more recently [18]. In particular, silica gel, obtained by polymerization of sodium silicates in acidic condition, constitutes an appropriate hydrophilic inorganic porous material to perform LDI analyses of polar biomolecules such as peptides as demonstrated by Zou et al. [13]. We chose standard porous materials usually used for chromatography, such as silica gel (acidic material, $\mathrm{pH} 6.5$ to 7.5 ) and alumina (basic material, $\mathrm{pH} 9.0$ to 10.5 ) presenting pore sizes of 60 and $90 \AA$, respectively. These commercially available chemicals are rather inexpensive and can be used without any specific treatment. Indeed, the same mass spectra were recovered with and without silica gel or alumina conditioning, which was initially performed to remove alkali metals that may be trapped in the material pores (exhaustive washings with hydrochloric acid $10 \%$ followed by deionized water). Besides, the chemical property of the material can be controlled. The presence of silanol groups on the silica gel surface that makes this support more hydrophilic than conventional DIOS plates can be capped by etherification with alkyl chains. Such hydrophobic grafted silica gel constitutes a standard stationary phase in the so-called reversed-phase liquid chromatography (REHPLC) and is also commercially available.

The present study was undertaken to simplify even further the sample preparation in DIOS-type mass spectrometry by using as inert matrix silica gel with specific chemical surface features (hydrophilic/hydrophobic) as well as alumina usually employed for chromatography (pore size of 60 and $90 \AA$, respectively). Such readily available low-cost material allowed fast and straightforward sample deposition on standard MALDI plates to conduct routine analyses carried out on any MALDI mass spectrometer. The results were compared with LDI data recorded from peptide samples directly deposited on the stainless steel plate without the use of any inorganic substrate. Analysis of peptide mixtures provided insight into desorption/ ionization mechanisms and highlighted the potential of such a mass spectrometry technique in proteomics.

\section{Experimental}

\section{Chemicals and Reagents}

All solvents were of analytical grade. Methanol was purchased from Fisher (Loughborough, UK), anhydrous dichloromethane and acetonitrile from Carlo Erba (Val de Reuil, France). The deionized water used in all the experiments was obtained using a Milli-Q system (Millipore, Milford, MA). Acetic acid was sup- plied by ProLabo (Fontenay sous bois, France). Hydrochloric acid (37\%) was obtained from VWR (Fontenay sous bois, France). Sodium iodide and $\alpha$-cyano-4-hydroxycinnamic acid were purchased from Fluka (Saint Quentin Fallavier, France). A peptide calibration standard kit was purchased from Bruker Daltonics (Breeman, Germany). Polyethylene glycols (PEGs) were used as aqueous solutions. PEG600 and PEG1000 were purchased from Janssen Chimica (Beerse, Belgium). PEG2000, PEG3350 and 18-crown-6 ether were obtained from Sigma Aldrich (Saint Quentin Fallavier, France). All porous material used in this study presented particle size of $0.063-0.200 \mathrm{~mm}$ (70-230 mesh ASTM). Silica gel Geduran Si 60 (pore size of $60 \AA$ ), aluminium oxide 60 (pore size of $60 \AA$ ), and aluminium oxide 90 (pore size of $90 \AA$ ) were purchased from Merck (Fontenay sous bois, France). Silica gel 100 (pore size of $100 \AA$ ) was purchased from Fluka. Reversed-phase silica gel was prepared according to standard procedure [21].

\section{Peptide Syntheses, Purification, and Storage}

Peptides were synthesized using the conventional methodology of solid-phase peptide synthesis using Fmoc chemistry [22] and purified by reversed-phase preparative HPLC. Each peptide under study was dissolved in a solution of water:acetonitrile (vol/vol, 1:1). These stock solutions at a concentration of $10^{-3} \mathrm{M}$ were stored in glass containers at $4{ }^{\circ} \mathrm{C}$ during the course of the studies.

\section{Cytochrome c Tryptic Digestion}

Protein (trypsin and cytochrome $c$ ) solutions at a concentration of $0.1 \mu \mathrm{g} / \mu \mathrm{L}$ in an ammonium bicarbonate buffer solution $\left(\mathrm{NH}_{4}^{+} \mathrm{HCO}_{3}^{-}\right.$at $50 \mathrm{mM}$, pH8 adjusted with $\mathrm{HCl} 12 \mathrm{~N}$ and $\mathrm{KOH} 5 \mathrm{~N}$ ) were prepared; $1 \mu \mathrm{L}$ of trypsin solution was added to $9 \mu \mathrm{L}$ of cytochrome $c$ solution and allowed to react at $37^{\circ} \mathrm{C}$ for $3 \mathrm{~h}$.

\section{Liquid Chromatography}

An Alliance 2690 (Waters, Milford, MA) HPLC system equipped with an autosampler was used to deliver the mobile phase, continuously degassed, at a flow rate of 1 $\mathrm{mL} / \mathrm{min}$ through the reversed-phase stationary phase Nucleosil 5C18 cartridge $(250 \times 4.6 \mathrm{~mm}$ i.d.) packed with $5 \mu \mathrm{m}$ particles (Macherey-Nagel, Düren, Germany). A linear gradient from $1 \%$ to $80 \%$ of acetonitrile in water containing $0.1 \%$ of acetic acid was applied in $120 \mathrm{~min}$. The percentage of acetonitrile was raised to $95 \%$ in $1 \mathrm{~min}$, maintained at $95 \%$ for $5 \mathrm{~min}$. The column was then brought back to the initial conditions in $1 \mathrm{~min}$ and equilibrated for $9 \mathrm{~min}$. The total run cycle was thus equal to $136 \mathrm{~min}$. In all experiments, $5 \mu \mathrm{L}$ of samples were injected at $25^{\circ} \mathrm{C}$. UV and mass spectrometric detections were simultaneously achieved. The mobile phase was first admitted into the UV cell of a PDA photodiode array detector (Waters) set up at $214 \mathrm{~nm}$ 
then split before the mass spectrometer entrance to reach $100 \mu \mathrm{L} / \mathrm{min}$ of eluent infused in the electrospray ionization (ESI) source. ESI mass spectra were recorded on a Quattro Micro triple quadrupole mass spectrometer (Waters). The mass spectrometer was calibrated in the positive ion mode using a mixture of $\mathrm{NaI}$ and CsI. Voltages were set at $+3.5 \mathrm{kV}$ for the capillary and +0.5 $\mathrm{kV}$ for the skimmer lens. The source was heated at $120^{\circ} \mathrm{C}$. Nitrogen was used as nebulizing and drying gas at $15 \mathrm{~L} / \mathrm{h}$ and $250 \mathrm{~L} / \mathrm{h}$, respectively. Mass spectrometric data were acquired in full scan mode. The sampling cone voltage was set up at $30 \mathrm{~V}$.

\section{Mass Spectrometry}

Mass spectra were acquired using the reflectron mode on an Ultraflex mass spectrometer (Bruker Daltonik $\mathrm{GmbH}$, Bremen, Germany). The serial instrument was equipped with a $337-\mathrm{nm} 50-\mathrm{Hz} \mathrm{N}_{2}$ laser used with a $33 \%$ attenuation level.

For MALDI-TOF mass spectra acquisition, the acceleration voltage was set to $25 \mathrm{kV}$, the pulsed extraction voltage to $21.85 \mathrm{kV}$, the extraction delay to $40 \mathrm{~ns}$, and the reflector voltage to $26,30 \mathrm{kV}$. The achieved peak resolution was between 3000 and 20,000, depending on the peaks. External calibrations were performed with a mixture of peptides (Bruker Daltonik $\mathrm{GmbH}$ ). One calibrant spot was deposited in the center of a 9-sample square and used to calibrate these samples (the overall distance between the calibration spot and the measured sample was always between 4 and $7 \mathrm{~mm}$ ). This procedure ensures reaching mass errors far below the $40 \mathrm{ppm}$ level.

For LDI mass spectra acquisition, the method described above was applied with two extraction delay values of $40 \mathrm{~ns}$ for masses ranging between 500 and 1000 Da or 100 ns for masses superior to 1000 Da. Peak resolution was between 2000 and 5000. Mass spectra were typically acquired from an average of 100 laser pulses. External calibrations were performed with a mixture of polyethylene glycols as mentioned above.

\section{Sample Preparation for MALDI Analysis}

The peptide stock solutions were diluted to reach concentrations of $10^{-6} \mathrm{M}$. The samples were first analyzed by MALDI mass spectrometry using $\alpha$-cyano-4- hydroxycinnamic acid (CHCA) as matrix [10 $\mathrm{mg} / \mathrm{mL}$ in water:acetonitrile ( $\mathrm{vol} / \mathrm{vol}, 70: 30)$ corresponding to a concentration of $5 \times 10^{-3} \mathrm{M}$ ]. Peptide and matrix solutions were mixed to achieve a $1 / 5000$ ratio; $1 \mu \mathrm{L}$ of the peptide/matrix mixture was deposited onto the MALDI plate and allowed to air-dry providing 1 pmol of peptide in each deposit. Acidified CHCA matrix solution containing $1 \%$ of acetic acid was also used.

The same sets of experiments were recorded for 2,5-dihydroxybenzoic acid (DHB) matrix dissolved in water/acetonitrile (50:50, vol/vol) or in water:acetonitrile $(50: 50, \mathrm{vol} / \mathrm{vol})$ containing $1 \%$ of acetic acid.

\section{Silica Gel Conditioning}

To remove any alkali metal trapped into the inorganic material, repeated washings with aqueous $\mathrm{HCl}$ solution $(10 \%)$ followed by deionized water were performed. The silica gel was then dried in an oven overnight.

\section{Sample Preparation for LDI Analysis}

Fifty milligrams of the inorganic material (silica gel, reversed-phase silica gel, or aluminium oxide) was first suspended either in water $(1 \mathrm{~mL})$ or in an aqueous solution of sodium iodide at a concentration of $10^{-3}$ or $10^{-5} \mathrm{M}(1 \mathrm{~mL})$. This suspension was thoroughly vortexed and rapidly aliquoted to deposit $1 \mu \mathrm{L}$ on the MALDI plate. Then, $1 \mu \mathrm{L}$ of the peptide solution at a concentration of either $10^{-6} \mathrm{M}$ (1 pmol deposited) or $10^{-5} \mathrm{M}$ (10 pmol deposited) was added on the top of the inorganic substrate droplet and such final deposit (total volume of $2 \mu \mathrm{L}$ ) was then air-dried. Pictures of silica gel, reversed-phase silica gel or aluminium oxide peptide deposits are displayed in Figure 1. For multiple on-target peptide deposition, the initial $2 \mu \mathrm{L}$ deposit was allowed to dry, and then another $1 \mu \mathrm{L}$ of the peptide solution was added and allowed to dry. These operations were repeated as much as needed.

\section{Results and Discussion}

Recent developments in methods and technologies for analysis of biological samples by MALDI mass spectrometry [23] emphasize the potential of LDI techniques such as DIOS. Among the analyzed molecules so far, we focused our attention on peptides and, in particular, on

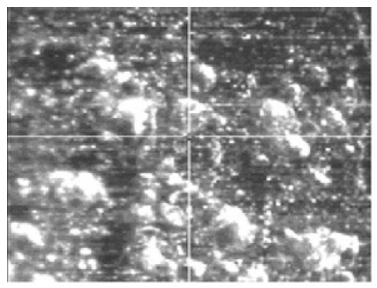

Silica gel

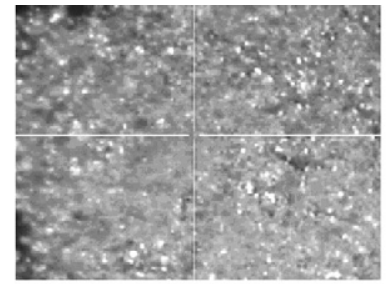

RP-Silica gel

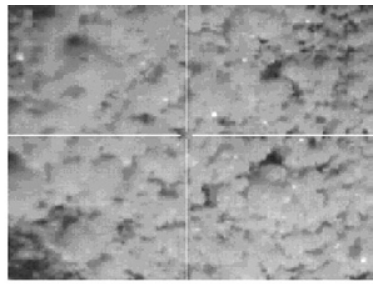

Alumina

Figure 1. Deposits of peptide/inorganic substrate mixture 
synthetic peptide mixtures to mimic peptide mass fingerprinting in proteomics. We therefore developed a straightforward LDI method based on the use of porous chromatography materials (silica gel, alumina) as an alternative to DIOS mass spectrometry for the analysis of peptides, and then we investigated the simultaneous detection of multiple peptides.

\section{Studied Peptides}

We synthesized 57 peptides to cover a large range of sequence diversity with masses ranging from $500 \mathrm{Da}(5$ residues) to $3000 \mathrm{Da}$ (25 residues) (Table 1, Figure 2). The hydrophobicity of the studied peptides was evaluated according to their chromatographic behavior on reversed-phase HPLC [24]. Most of the synthesized peptides possessed arginine or lysine at the C-terminal position that mimic tryptic peptides. However, the nature of any amino acid in that C-terminal position was also evaluated to take into account the detection of peptides issued from the C-terminal part of the protein. Besides, peptide chains terminated by a C-terminal carboxylic acid as well as a C-terminal amide were also prepared to mimic the proteolysis of naturally occurring amidated proteins $[25,26]$.

\section{Preliminary LDI Analyses}

The initial purpose of our study was to compare a "matrix-free" LDI technique with the most popular LDI method used in biomolecule identification, i.e., MALDI. Taking into account the pioneering works of several research groups in the early 1980s [1-9], which investigated direct laser desorption/ionization of organic materials, we first tried to analyze our peptide samples without any substrate by depositing the peptide solution directly on the stainless steel MALDI plate. The results were very promising in terms of sensitivity and detection mass range but the low mass part of the spectra was exhibiting a lot of signals (Figure 3). We thought that fragmentations were occurring due to the rather high laser fluence required to record signals. Under such conditions, we decided to use an inorganic material to improve the desorption/ionization process. Readily available low-cost silica gel and alumina were chosen, our LDI technique being thus related to DIOS.

\section{Silica Gel Versus Porous Silicon as LDI Substrate}

To simplify DIOS analyses and to make this technique readily available to MALDI mass spectrometry users, silica gel, reversed-phase silica gel, and alumina were investigated as inorganic matrix in LDI mass spectrometry. The sample work-up and the deposition procedure onto the standard MALDI plate were carefully examined before laser desorption/ionization of pure or mixed peptides investigations.

\section{Sample Deposition Protocols on MALDI Plates}

Several sample preparation methods were tested. First, the studied peptides were all dissolved in methanol at $10^{-3}$ to $10^{-4} \mathrm{M}$ concentrations. Methanol was primarily chosen for its high solubilization property and for its low boiling point. Silica gel (50 mg in $1 \mathrm{~mL}$ of solvent) was added to the methanolic peptide solution and the suspension obtained was vortexed before methanol elimination under vacuum. Under these conditions, peptide should not only be adsorbed onto the material surface but also trapped into the pores. The resulting silica gel was then deposited onto a double-sided adhesive tape fixed on the MALDI plate. The same protocol was also performed by using a solution of sodium iodide in methanol to induce cationization during sample ionization. Samples prepared using these methods gave no ion when irradiated. Thus, a step was added to the aforementioned procedures. Instead of fixing the treated silica gel onto an adhesive tape, the material was suspended again either in an aqueous or a methanolic solution of sodium iodide. A droplet of such a suspension was deposited directly onto the MALDI plate and air-dried. In contrast to the first methods, samples deposited using these on-target "wet" silica gel protocols produced the expected ions when irradiated. Such results indicated that trapping the peptides in the silica gel pores is not sufficient (solid deposit). A liquid phase is required during sample preparation, probably to properly swell the inorganic porous material on the MALDI plate, thus facilitating the subsequent LDI process. Based on these results, and to simplify the sample preparation, neat silica gel was first suspended either in water or in an aqueous solution of sodium iodide and a droplet of the suspension was deposited on the MALDI plate. Then, a droplet of the peptide solution was added on the top and the deposit was then air-dried. The order of the deposit can also be inverted. The fact that there was no solvent elimination step allowed more flexibility in the choice of the solvent used to solubilize the studied analytes. Protic solvents (methanol, water:acetonitrile (1:1, vol/vol), water:acetonitrile $(1: 1, \mathrm{vol} / \mathrm{vol})$ containing $1 \%$ of trifluoroacetic acid) as well as aprotic solvents (acetonitrile, dichloromethane) were successfully applied. These two last procedures involving on-target "wet" peptide/porous material (silica gel, reversed-phase silica gel, or alumina) mixing were used throughout the study. Although the silica gel pore size was reported to be critical for ion detection (with a minimum of $100 \AA$ required) [13], we found that smaller pores were suitable provided that the porous material was properly swollen on the MALDI probe (Figure 1). Similar results were observed by Wysocki and coworkers [18] with silicon nanopowders of 5, 30, and $50 \mathrm{~nm}$ particle size, the 50 $\mathrm{nm}$ particles giving the lowest analyte signal intensity. Thus, in addition to the pore size, the porous material surface wet-ability seemed also to be a very important parameter to perform successful LDI analyses. 


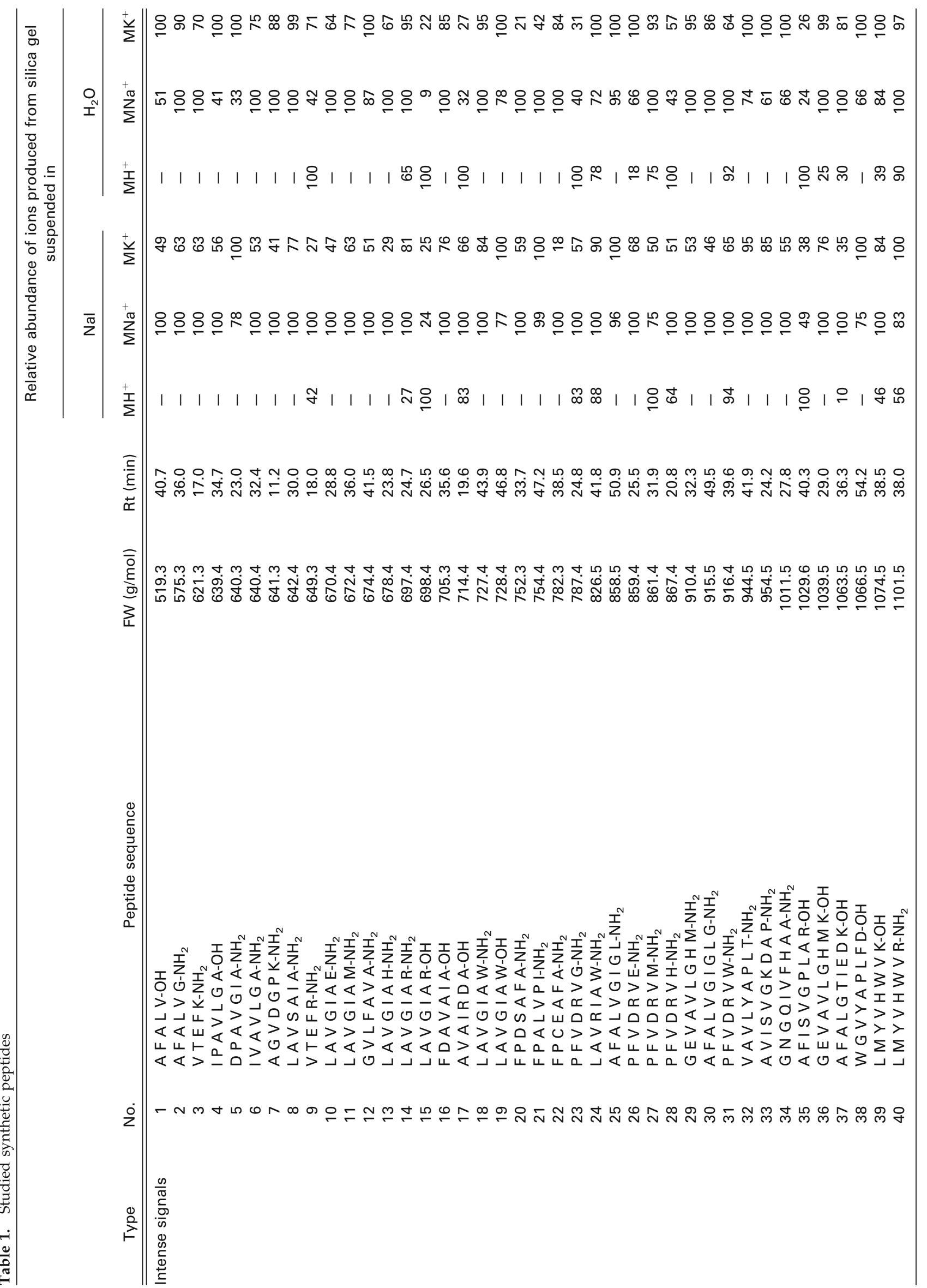




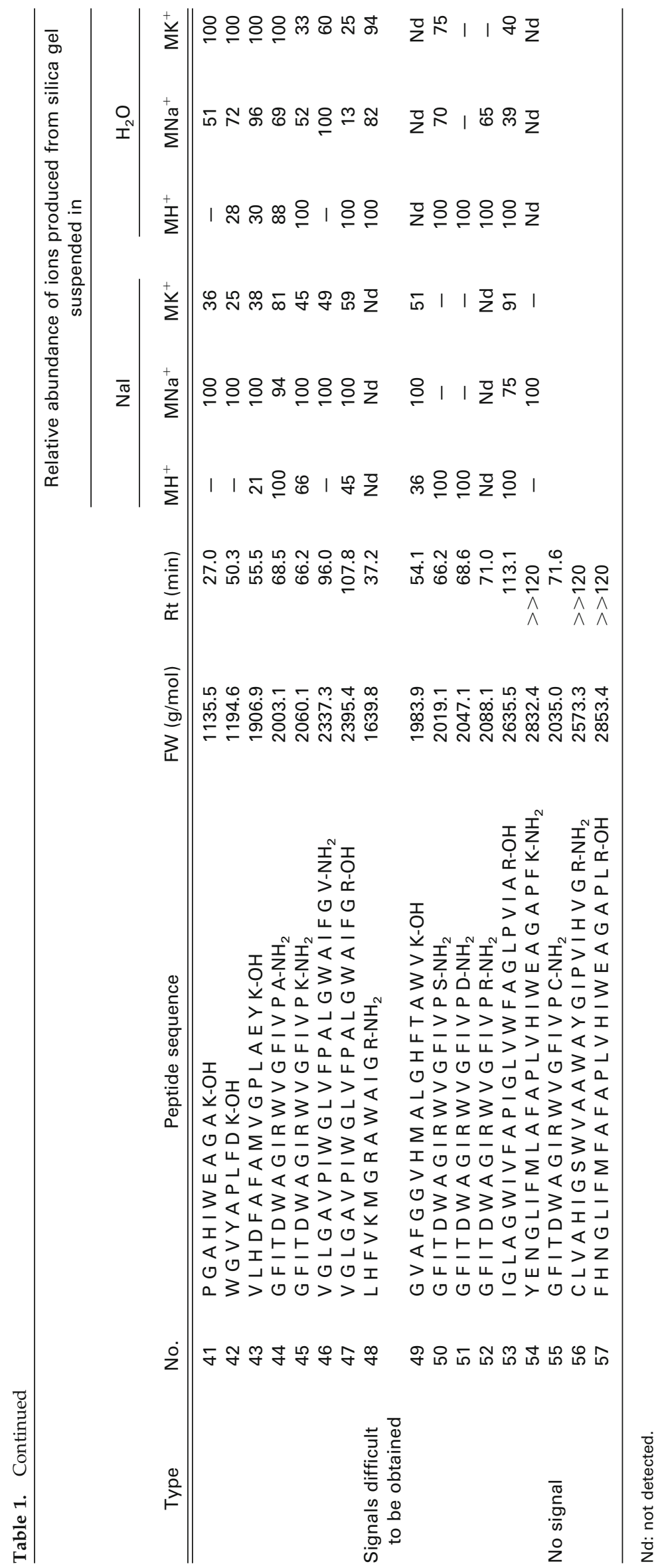




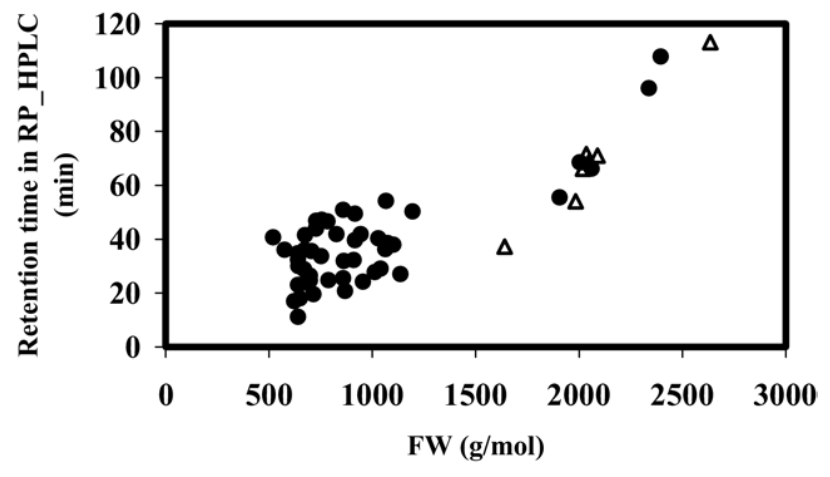

- Detected peptides

$\Delta$ Undetected peptides or peptides difficult to detect

Figure 2. Peptide detection in LDI on silica gel.

\section{Investigation of LDI Process}

Each peptide solution was individually analyzed by conventional MALDI mass spectrometry and by LDI method using silica gel and alumina as inorganic matrix. It has been observed that source parameters needed to be modified for switching from MALDI to LDI: higher laser fluences as well as increased extraction delays were required to produce signals that were even less intense with the latter technique. To ascertain the method sensitivity, the limit of detection in LDI mass spectrometry of peptides adsorbed on silica gel was investigated. The same samples used for MALDI at the concentration level of $10^{-6} \mathrm{M}$ (1 pmol of deposited peptide) were tested. The results were not good for all peptides: some samples were exhibiting mass spectra with well-defined signals (S/N around 10) but other peptides did not provide any expected ions. In such case, multiple depositions of the peptide solution on the same spot were performed (allowing each deposit to dry before the next one). Such on target sample concentration allowed to increase the peptide amount and provided the expected mass spectra. So, since the LDI response was peptide-dependent at the concentration level of $10^{-6} \mathrm{M}$, we increased the concentration by a factor 10. Just keeping under consideration the quality of the recorded mass spectra by measuring the $\mathrm{S} / \mathrm{N}$ ratio, we found that all samples with a peptide concentration of $10^{-5} \mathrm{M}$ gave very correct mass spectra (the worst response showed $\mathrm{S} / \mathrm{N}=19$ ). Figure 3 displays the LDI and MALDI mass spectra of peptide no. 2, AFALVG-NH $\mathrm{N}_{2}$, recorded from silica gel, alumina, DHB matrix, and the stainless steel surface. Another example is provided in the Supporting Information (which can be found in the electronic version of this article) for a high molecular weight sequence (peptide no. 46, VGLGADPIWGLVFPALGWAIFGV-NH ${ }_{2}$ ). At this concentration level $(10 \times$ additional concentration than the one

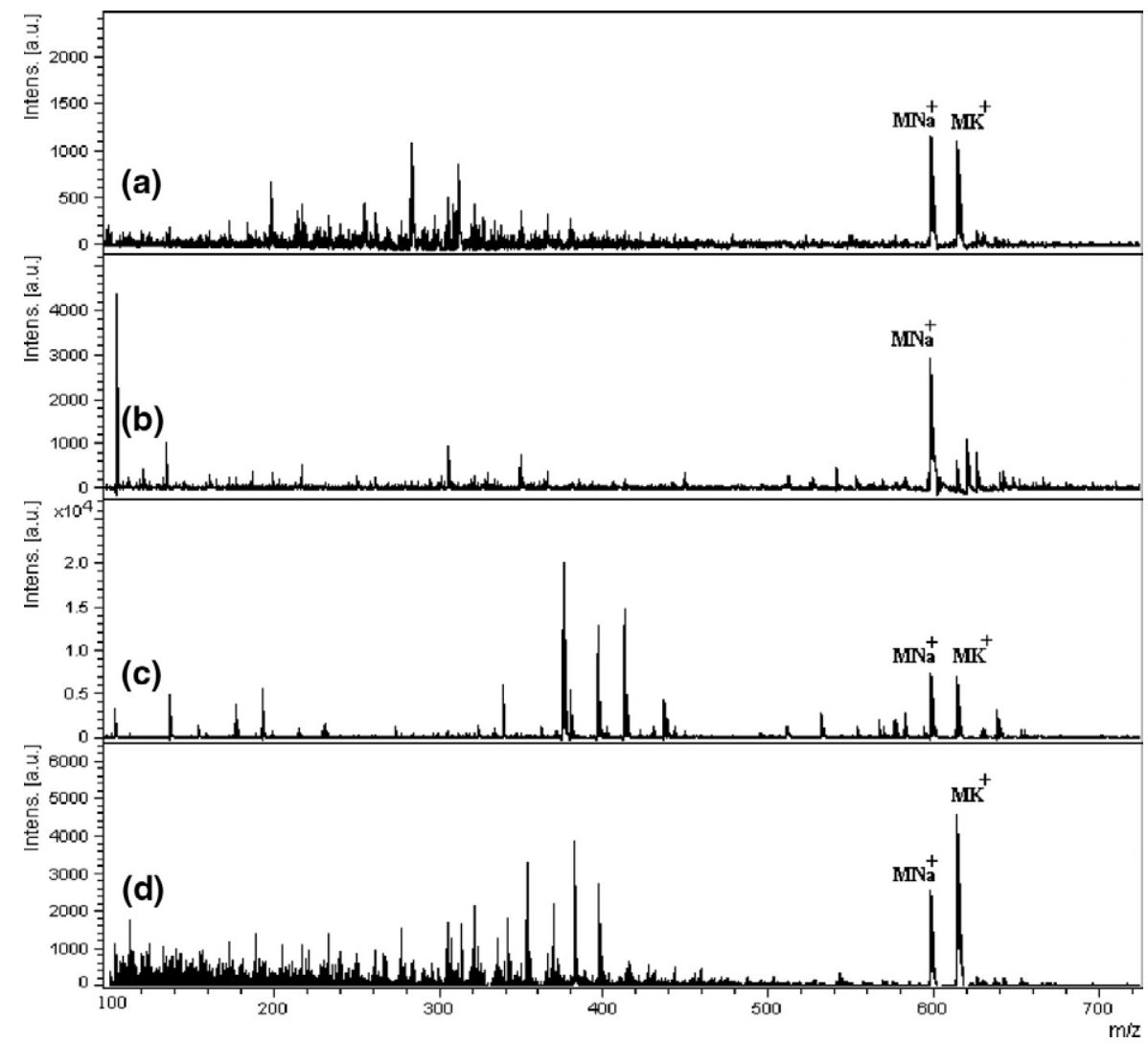

Figure 3. LDI and MALDI mass spectra of peptide no. 2 (AFALVG-NH ${ }_{2}$ ) acquired from (a) silica gel, (b) alumina, (c) DHB matrix, (d) stainless steel surface. 
Table 2. Statistical evaluation of the reproducibility of LDI on silica gel

\begin{tabular}{|c|c|c|c|}
\hline \multirow[b]{2}{*}{ Peptide no. } & \multirow[b]{2}{*}{ Cationized species } & \multicolumn{2}{|c|}{$\begin{array}{c}\text { Abundance of cationized } \\
\text { species }\end{array}$} \\
\hline & & Mean value & Deviation \\
\hline \multirow[t]{2}{*}{46} & $\mathrm{Na}^{+}$ & 357.0 & 15.95 \\
\hline & $\mathrm{K}^{+}$ & 433.6 & 48.15 \\
\hline \multirow[t]{2}{*}{40} & $\mathrm{Na}^{+}$ & 299.9 & 115.41 \\
\hline & $\mathrm{K}^{+}$ & 420.4 & 98.72 \\
\hline \multirow[t]{2}{*}{30} & $\mathrm{Na}^{+}$ & 2692.4 & 248.39 \\
\hline & $\mathrm{K}^{+}$ & 2164.0 & 83.65 \\
\hline \multirow[t]{2}{*}{11} & $\mathrm{Na}^{+}$ & 1852.2 & 362.71 \\
\hline & $\mathrm{K}^{+}$ & 1014.0 & 670.29 \\
\hline \multirow[t]{2}{*}{18} & $\mathrm{Na}^{+}$ & 1202.0 & 205.30 \\
\hline & $\mathrm{K}^{+}$ & 1129.2 & 43.59 \\
\hline \multirow[t]{2}{*}{14} & $\mathrm{Na}^{+}$ & 545.1 & 82.62 \\
\hline & $\mathrm{K}^{+}$ & 763.8 & 198.56 \\
\hline \multirow[t]{2}{*}{21} & $\mathrm{Na}^{+}$ & 1153.2 & 158.15 \\
\hline & $\mathrm{K}^{+}$ & 1544.1 & 174.08 \\
\hline \multirow[t]{2}{*}{25} & $\mathrm{Na}^{+}$ & 7115.8 & 718.09 \\
\hline & $\mathrm{K}^{+}$ & 9444.9 & 882.20 \\
\hline \multirow[t]{2}{*}{2} & $\mathrm{Na}^{+}$ & 3603.5 & 187.12 \\
\hline & $\mathrm{K}^{+}$ & 3602.7 & 237.38 \\
\hline \multirow[t]{2}{*}{12} & $\mathrm{Na}^{+}$ & 2454.3 & 180.76 \\
\hline & $\mathrm{K}^{+}$ & 2826.2 & 452.24 \\
\hline
\end{tabular}

used in MALDI corresponding to $10 \mathrm{pmol}$ of peptide in each deposit), the described method was validated by statistical evaluation of reproducibility. Ten peptides at a concentration of $10^{-5} \mathrm{M}$ (10 pmol deposited for each LDI experiment) with molecular mass ranging from 575 to $2337 \mathrm{~g} / \mathrm{mol}$ were selected for this study. Each sample was deposited five times and analyzed under the same experimental conditions (laser fluence, number of laser shots). The abundance of each cationized ion $\left(\mathrm{MNa}^{+}\right.$ and $\mathrm{MK}^{+}$) was measured; the variation around the mean value is reported in Table 2 .

It should be noted that the low molecular mass range of the recorded LDI mass spectra was not totally free of chemical noise. Pollution from chemicals used during the manufacture of the material [18] or during the material storage [27] was envisaged. Extensive washing and drying under vacuum of the silica gel before its usage surprisingly did not allow us to solve this problem.

Above all, the LDI mass spectra were dominated by sodiated species, protonation, and cationization with potassium being also frequently observed as shown in Table 1. The peptide signals were thus spread over three ions affecting the overall detection sensitivity (lower $\mathrm{S} / \mathrm{N}$ ratio for each ion). Although all peptides that were prepared by solid-phase procedures have been purified by preparative LC (experimental data supplied in the Supporting Information), they exhibited MALDI and ESI MS data already displaying both $\mathrm{MH}^{+}$ and $\mathrm{MNa}^{+}$ions. Moreover, the peptides were stored as stock solutions in glass containers for several weeks, which increased the alkali metal contamination significantly. Thus, MALDI mass spectra that were recorded to check sample integrity before LDI analyses already showed an increased ratio of cationization versus protonation (MALDI data shown in Figure 3). At that stage, standard cleanup steps to remove alkali metals were undertaken, such as ZipTip (Millipore, Saint Quentin en Yvelines, France) filtration, on MALDI target washing with ammonium citrate solution, but none of these protocols provided satisfactory results. Most of the time, the salt content was barely decreased and few peptides were even lost during the ammonium citrate washing. Using acidified matrix solution or acidified peptide solution did not provide any improvement (data provided in the Supporting Information). The best desalting method we found was to trap the alkali metal in situ in the deposit with an appropriate scavenger such as crown ethers (supporting information). Moreover, for LDI experiments on porous substrates, silica gel and alumina contamination by alkali metals could not be completely ruled out. A specific washing protocol with hydrochloric acid was used to prevent such substrate contamination, but the results in terms of cationization were similar with and without porous material acidic treatment. In an attempt to homogenize the LDI response, two strategies were pursued: (1) pushing the cationization to completion or (2) suppressing the alkali metals. The former protocol that implied addition of $\mathrm{NaI}$ to the aqueous silica gel suspension did not succeed since the cationized signal was enhanced but the protonated peptide, when present, remained. When sodium iodide was replaced by potassium iodide, the recorded LDI mass spectra exhibited both $(\mathrm{M}+\mathrm{Na})^{+}$and $(\mathrm{M}+\mathrm{K})^{+}$species. On the other hand, washing the silica gel with a solution of hydrochloric acid $(10 \%)$ to remove the alkali metals before sample preparation was not sufficient to eliminate the cationized ions. Crown ether 18-C-6, an alkali metal scavenger [28], was mixed with the peptide/silica gel suspension in an attempt to enhance production of protonated ions upon laser irradiation. Results indicated that the method was successful since the production of ions was more homogeneous than previously observed. Indeed, in most cases, both cationized species $\left(\mathrm{MNa}^{+}\right.$and $\left.\mathrm{MK}^{+}\right)$ were greatly diminished with the protonated ion representing the base peak in most of the recorded LDI mass spectra (supporting information).

Among all studied peptide sequences, three categories can be distinguished according to the production of molecular ions as displayed in Table 1 . When testing the reproducibility of the method at the concentration level of $10^{-6} \mathrm{M}$, some peptides gave abundant ions in all experiments ( $80 \%$ of the studied peptides, mostly small sequences) as depicted in Figure 2 by black dots. On the contrary, some of them failed to be ionized (5\%, medium to high masses). In between, in few cases, the LDI process was quite difficult to reproduce $(15 \%$, medium to high masses). These peptides are represented by open triangles in Figure 2. Although the DIOS technique is restricted to the analysis of rather low molecular weight compounds, correlation of ionization out- 


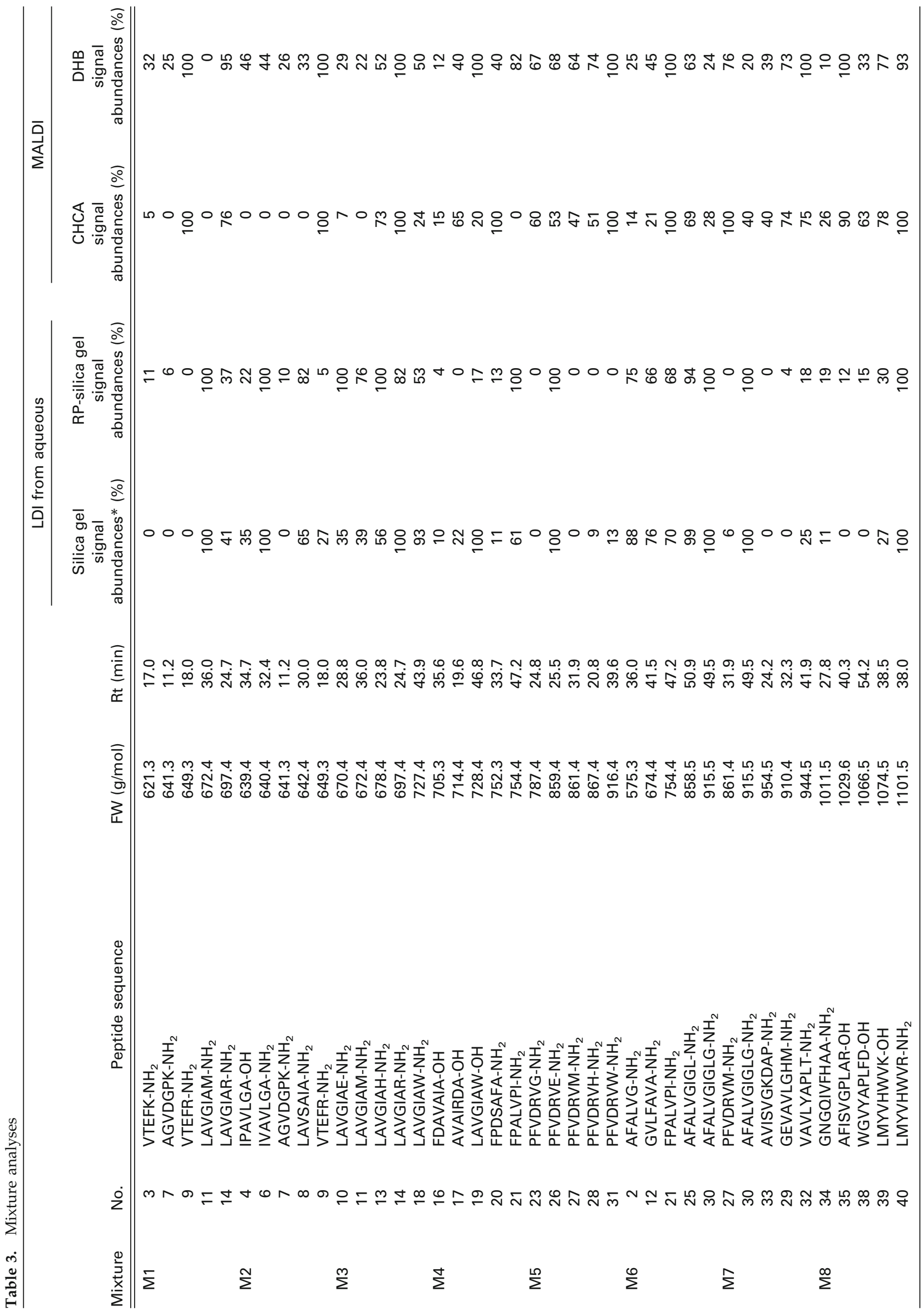




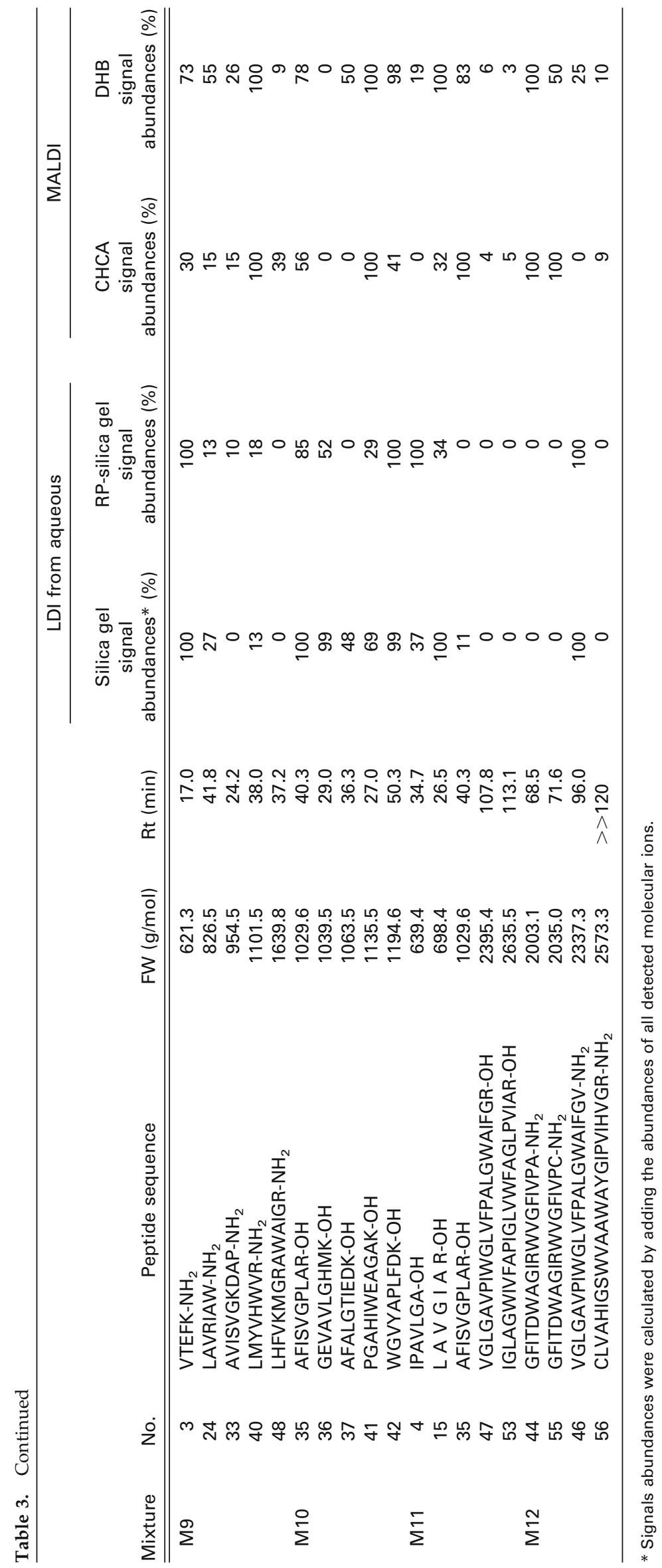


put and peptide molecular mass was not satisfactory since few medium size peptides were not detected, whereas some long chains were observed. So, the influence of compound hydrophobicity on the laser desorption/ionization on silica gel process was considered. In that sense, reversed-phase silica gel was used for a second round of peptide analyses. No major differences were observed between the two sets of analyses. The samples that failed to exhibit ions upon laser irradiation were mostly responding similarly when adsorbed onto reversed-phase silica. Moreover, peptides that were successfully analyzed from silica gel, mainly as (M + $\mathrm{Na})^{+}$ions, were also detected as such from reversedphase silica material but with lower abundances. Further studies were undertaken by varying the suspending solvent nature (protic/aprotic), by adding a cationizing agent $(\mathrm{NaI})$, but the mass spectra were merely not changed. Lastly, tuning from acidic silica gel to basic alumina while keeping the same pore size (60 $\AA$ ) provided the same performances in terms of sensitivity, resolution, and nature of produced ions $\left(\mathrm{MNa}^{+}\right)$ but a lower mass cut-off for peptide detection was observed (around $1000 \mathrm{Da}$ ). No improvement was obtained from alumina suspended in aqueous sodium iodide. One explanation of such a lower quality response could come from the fact that alumina was not swollen in water.

Finally, the pore size was investigated. All experiments were duplicated from silica gel and alumina presenting larger pores (100 and $90 \AA$, respectively). As previously described, cationization with sodium and to a lesser extent with potassium was mainly occurring but surprisingly, the same mass cut-off for peptide detection was observed for each porous material.

These results indicated that the chemical nature of the inert surface (acidic or basic, hydrophilic or hydrophobic) was apparently not playing a role in the desorption/ionization output. The material physical prop- erties seemed to be more important. According to the described results, mass range detection and the sensitivity were apparently not only governed by the material pore size. The capacity of the material to properly swell in the solubilizing/deposition solvent (surface wet-ability) must be considered. Finding the right combination between the material, the solvent and the deposit protocol seems prerequisite for successful analyses. Furthermore, results obtained from LDI and LDI with samples doped by the chosen inorganic materials were very similar (Figure 3 and supporting information). The same sensitivity and the same mass range were observed for signal detection for experiments conducted simultaneously on the same steel plate with and without silica gel mixed with the peptide solution. The major difference was the laser fluence that was very high in LDI. Fragmentations that could be triggered under such harsh condition led to crowded mass spectra in the low mass range. Besides, data acquisition was more difficult: getting signals from peptide solutions dried on the stainless steel surface was sometimes more tricky, and thus less reproducible, compared with peptide solutions that were dried with silica gel, the peptide being certainly trapped inside the support pores and targeted more efficiently for laser irradiation.

\section{Mixtures of Peptides}

The mechanisms of desorption/ionization are different in DIOS and MALDI mass spectrometry [29]. While the desorption of organic compounds that are trapped into porous silicon is seemingly induced by local surface heating [10], it would be interesting to compare the discrimination for the same peptide mixtures observed using LDI and MALDI. Two different matrices (CHCA and $\mathrm{DHB}$ ) were investigated for MALDI analyses. Twelve mixtures containing a maximum of five pep-
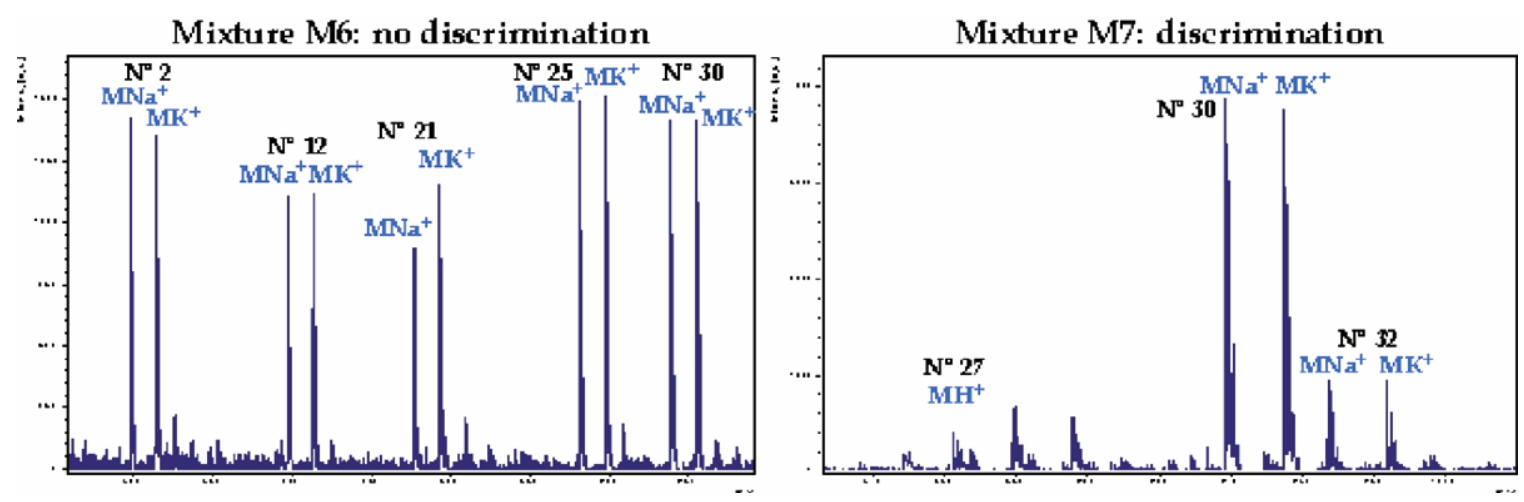

Figure 4. LDI analyses from silica gel of two mixtures composed each of equimolar amounts of five peptides. Mixture M6, peptide no. 2 (AFALVG-NH $2,575.3 \mathrm{~g} / \mathrm{mol})$, peptide no.12 (GVLFAVA-NH ${ }_{2}$, $674.4 \mathrm{~g} / \mathrm{mol}$ ), peptide no. 21 (FPALVPI-NH ${ }_{2}, 754.4 \mathrm{~g} / \mathrm{mol}$ ), peptide no. 25 (AFALVGIGL-NH ${ }_{2}, 858.5$ $\mathrm{g} / \mathrm{mol}$ ), and peptide no. 30 (AFALVGIGLG-NH $\mathrm{N}_{2} 915.5 \mathrm{~g} / \mathrm{mol}$ ). Mixture M7, peptide no. 27 (PFVDRVM-NH ${ }_{2}, 861.4 \mathrm{~g} / \mathrm{mol}$ ), peptide no. 30 (AFALVGIGLG-NH ${ }_{2}, 915.5 \mathrm{~g} / \mathrm{mol}$ ), peptide no. 33 (AVISVGKDAP-NH $2,954.5 \mathrm{~g} / \mathrm{mol})$, peptide no. 29 (GEVAVLGHM-NH ${ }_{2}, 910.4 \mathrm{~g} / \mathrm{mol}$ ), and peptide no. 32 (VAVLYAPLT- $\mathrm{NH}_{2}, 944.5 \mathrm{~g} / \mathrm{mol}$ ). 
tides previously analyzed individually (Table 3) were designed to cover the following situations:

- Peptide sequences of close molecular masses but with different amino acid compositions;

- peptides mainly constituted by hydrophobic or basic residues but of variable length;

- peptides differing only by the nature of the C-terminal amino acid;

- peptides differing only by the nature of the C-terminal end, a carboxylic acid or an amide function.

Two situations were encountered during the LDI analyses: (1) peptides all responded similarly or, on the contrary, (2) some peptides were under- or overexpressed (Figure 4). The same behaviors were recovered from LDI analyses performed with reversed-phase silica gel and alumina, the discrimination being even more important in the latter case. Moreover, wet de- posit containing or not containing a cationizing agent yielded similar discrimination, indicating that competitions for proton and for alkali metal attachment were thus similar. When an organic matrix was involved in the competitive ionization processes in MALDI experiments, peptide discrimination was also evidenced (Table 3) but remarkably, in most cases, the peptides that were exhibiting the most abundant signals in LDI on porous silica gel were underexpressed in MALDI. No obvious correlation of discrimination with peptide hydrophobicity was provided.

\section{Tryptic Digest of Cytochrome c}

To illustrate the application of the described LDI on silica gel method to proteomics, the tryptic digest of cytochrome $c$ (Equus caballus) was analyzed. To keep the experimental conditions similar with the one used for model peptide analyses, the digest solution was depos-

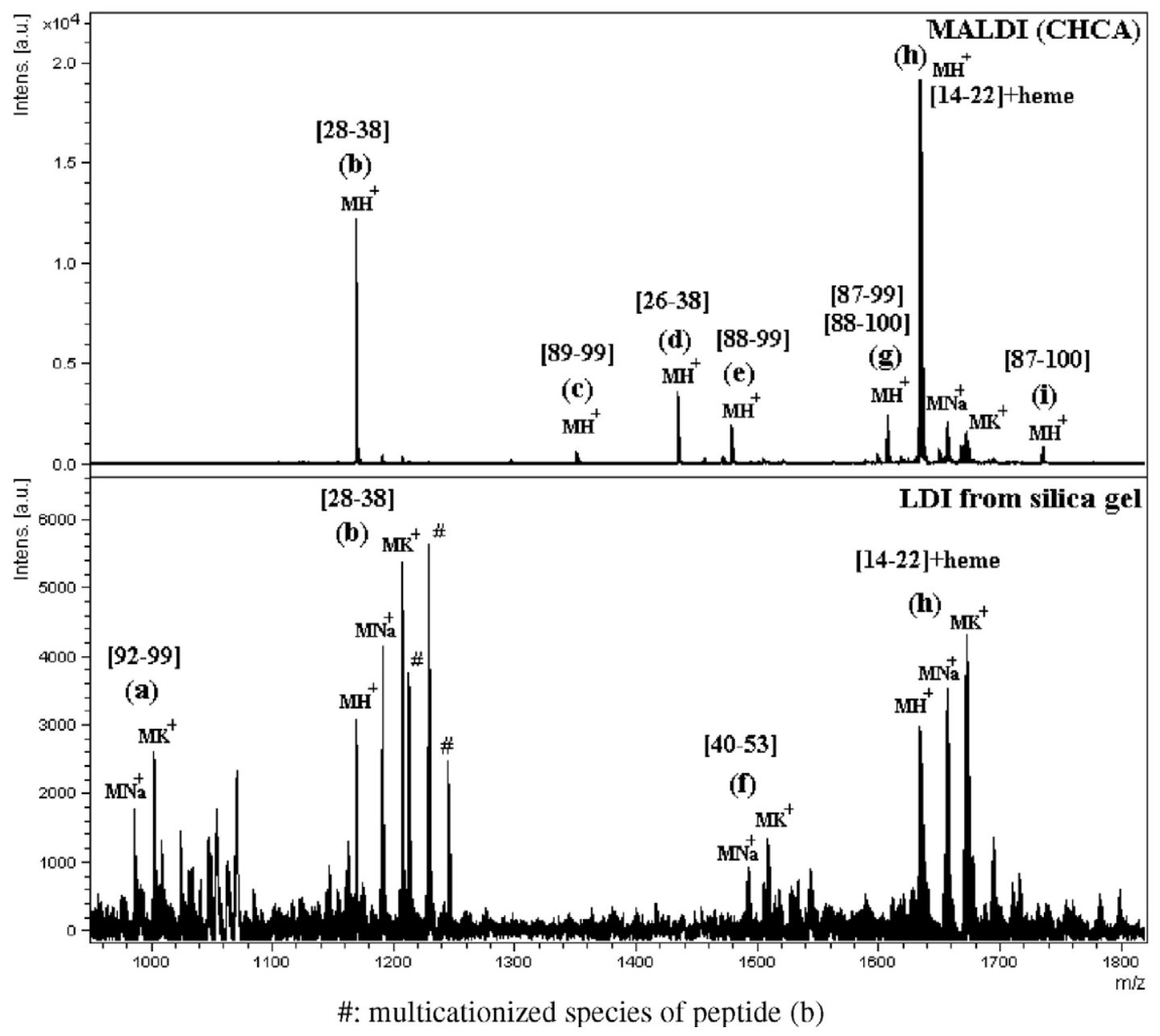

Figure 5. LDI from silica gel and MALDI mass spectra of tryptic cytochrome $c$ digest. Peptide (a): [92-99] (EDLIAYLK, $m / z$ 964.5), peptide (b): [28-38] (TGPNLHGLFGR, $m / z$ 1168.6), peptide (c): [89-99] (TEREDLIAYLK, m/z 1350.7), peptide (d): [26-38] (HKTGPNLHGLFGR, $m / z$ 1433.8), peptide (e): [88-99] (KTEREDLIAYLK, $m / z$ 1478.8), peptide (f): [40-53] (TGQAPGFTYTDANK, $m / z$ 1470.7), peptide (g): [87-99]/[88-100] (KKTEREDLIAYLK/KTEREDLIAYLKK, m/z 1606.9), peptide (h): [1422] + heme (CAQCHTVEK + heme, $m / z$ 1634.6) and peptide (i): [87-100] (KKTEREDLIAYLKK, 1735.2). 
ited as such without dilution (sample concentration around $10^{-6} \mathrm{M}$ ) and without desalting. The LDI mass spectrum recorded from silica gel doped digest solution is reproduced in Figure 5 and compared with the MALDI mass spectrum. Peptides were mainly detected in LDI as cationized species. Two peptides (compounds denoted $b$ and $h$ in Figure 5) were observed both in MALDI and LDI on silica gel. The other tryptic sequences were either only detected in MALDI mass spectrometry (compounds denoted c, d, e, g, i in Figure 5 ) or in LDI on silica gel (compounds denoted $a, f$ in Figure 5). Furthermore, the low mass range of the LDI mass spectrum showed the protonated ion of the heme together with four other tryptic peptide signals of low masses (data shown in the supporting information). This result indicates that LDI on silica gel and MALDI mass spectrometry are complementary techniques for the analysis of peptide mixtures.

\section{Conclusions}

The LDI mass spectrometry of peptides mixed with commercially available porous silica gel or alumina was readily achieved with standard MALDI instrument. Very easy sample preparation not requiring material conditioning or chemical derivatization was developed. The method was validated for a large panel of peptide sequences, confirming that not only the pore size but above all the "wet" environment of the trapped molecules is critical for successful analyses. Despite the difficulty to probe the origin of the detected ions (solution- or gas-phase reactions), discriminations evidenced during the competitive desorption/ionization of peptides illustrated the influence of the amino acid sequence on the mass spectrometry analysis hampering sometimes equal compound detection and resulting in biased mixture screening. These facts are worth considering in proteomics. Further studies are thus under investigation to check the method as an alternative to MALDI mass spectrometry for peptide mass fingerprinting of tryptic digests with better low mass detection and overall less information loss.

\section{Acknowledgments}

The authors thank Dr. Laetitia Mouls and Dr. Pascal Verdié for providing some peptides and David Paramelle for performing the cytochrome $c$ tryptic digestion.

\section{References}

1. Posthumus, M. A.; Kistemaker, P. G.; Meuzelaar, H. L. C.; Ten Noever de Brauw, M. C. Laser Desorption-Mass Spectrometry of Polar Nonvolatile Bio-Organic Molecules. Anal. Chem. 1978, 50, 985-991.

2. Zakett, D.; Schoen, A. E.; Cooks, R. G.; Hemberger, P. H. LaserDesorption Mass Spectrometry/Mass Spectrometry and the Mechanism of Desorption Ionization. J. Am. Chem. Soc. 1981, 103, 1295-1297.
3. Hardin, E. D.; Vestal, M. L. Laser Ionization Mass Spectrometry of Nonvolatile Samples. Anal. Chem. 1981, 53, 1492-1497.

4. Busch, K. L.; Cooks, R. G. Mass Spectrometry of Large, Fragile, and Involatile Molecules. Science 1982, 218, 247-254.

5. Hercules, D. M. Organic Mass Spectrometry Using the Laser Microprobe. Pure Appl. Chem. 1983, 55, 1869-1885.

6. Tabet, J.-C.; Cotter, R. J. Laser Desorption Time-of-Flight Mass Spectrometry of High Mass Molecules. Anal. Chem. 1984, 56, 1662-1667.

7. Sundqvist, B. U. R. Desorption Methods in Mass Spectrometry. Int. J. Mass Spectrom. Ion Processes 1992, 118/119, 265-287.

8. Busch, K. L. Desorption Ionization Mass Spectrometry. J. Mass Spectrom. 1995, 30, 233-240.

9. Zenobi, R. In Situ Analysis of Surfaces and Mixtures by Laser Desorption Mass Spectrometry. Int. J. Mass Spectrom. Ion Processes 1995, 145, 51-77.

10. Wei, J.; Buriak, J.; Siuzdak, G. Desorption-Ionization Mass Spectrometry on Porous Silicon. Nature 1999, 399, 243-246.

11. Lewis, W. G.; Shen, Z.; Finn, M. G.; Siuzdak, G. Desorption/Ionization on Silicon (DIOS) Mass Spectrometry: Background and Applications. Int. J. Mass Spectrom. 2003, 226, 107-116.

12. Vaidyanathan, S.; Jones, D.; Ellis, J.; Jenkins, T.; Chong, C.; Anderson, M.; Goodacre, R. Laser Desorption/Ionization Mass Spectrometry on Porous Silicon for Metabolome Analyses: Influence of Surface Oxidation. Rapid Commun. Mass Spectrom. 2007, 21, 2157-2166.

13. Zhang, Q.; Zou, H.; Guo, Z.; Zhang, Q.; Chen, X.; Ni, J. Matrix-Assisted Laser Desorption/Ionization Mass Spectrometry Using Porous Silicon and Silica Gel as Matrix. Rapid Commun. Mass Spectrom. 2001, 15, 217-223.

14. Budimir, N.; Blais, J.-C.; Fournier, F.; Tabet, J.-C. Desorption/Ionization on Porous Silicon Mass Spectrometry (DIOS) of Model Cationized Fatty Acids. J. Mass Spectrom. 2007, 42, 42-48.

15. Xu, S.; Li, Y.; Zou, H.; Qiu, J.; Guo, Z.; Guo, B. Carbon Nanotubes as Assisted Matrix for Laser Desorption/Ionization Time-of-Flight Mass Spectrometry. Anal. Chem. 2003, 75, 6191-6195.

16. Ugarov, M. V.; Egan, T.; Khabashesku, D. V.; Schultz, J. A.; Peng, H.; Khabashesku, V. N.; Furutani, H.; Prather, K. S.; Wang, H.-W. J.; Jackson, S. N.; Woods, A. S. MALDI Matrices for biomolecular Analysis Based on Functionalized Carbon Nanomaterials. Anal. Chem. 2004, 76, 6734-6742.

17. Mclean, J. A.; Stumpo, K. A.; Russell, D. H. Size-Selected (2-10 nm) Gold Nanoparticles for Matrix-Assisted Laser Desorption Ionization of Peptides. J. Am. Chem. Soc. 2005, 127, 5304-5305.

18. Wen, X.; Dagan, S.; Wysocki, V. H. Small-Molecule Analysis with Silicon Nanoparticles-Assisted Laser Desorption/Ionization Mass Spectrometry. Anal. Chem. 2007, 79, 434-444.

19. Nayak, R.; Knapp, D. R. Effects of Thin-Film Structural Parameters on Laser Desorption/Ionization from Porous Alumina. Anal. Chem. 2007 $79,4950-4956$.

20. Thomas, J. J.; Shen, Z.; Crowell, J. E.; Finn, M. G.; Siuzdak, G Desorption/Ionization on silicon (DIOS): A Diverse Mass Spectrometry Platform for Protein Characterization. PNAS 2001, 98, 4932-4937.

21. Yu, X.; Zhao, R.; Liu, G. Q. A Novel Method for the Preparation of C[18] Ester-Bonded RP-HPLC Packings. Chromatographia 2000, 52, 517-519.

22. Stewart, J. M.; Young, J. D. Solid Phase Peptide Synthesis, 2nd ed.; Pierce Chemical Company: Rockford, IL, 1984.

23. Pan, C.; Xu, S.; Zhou, H.; Fu, Y.; Ye, M.; Zou, H. Recent Developments in Methods and Technology for Analysis of Biological Sample by MALDI-TOF-MS. Anal. Biochem. 2007, 387, 193-204.

24. Krokhin, O. V.; Ying, S.; Cortens, J. P.; Ghosh, D.; Spicer, V.; Ens, W.; Standing, K. G.; Beavis, R. C.; Wilkins, J. A. Use of Peptide Retention Time Prediction for Protein Identification by Off-Line Reversed-Phase HPLC-MALDI MS/MS. Anal. Chem. 2006, 78, 6265-6269.

25. Mouls, L.; Subra, G.; Aubagnac, J.-L.; Martinez, J.; Enjalbal, C. Tandem Mass Spectrometry of Amidated Peptides. J. Mass Spectrom. 2006, 41, 1470-1483.

26. Mouls, L.; Aubagnac, J.-L.; Martinez, J.; Enjalbal, C. Low Energy Peptide Fragmentations in an ESI-Q-TOF Type Mass Spectrometer. J. Proteome Res. 2007, 6, 1378-1391.

27. Shen, Z.; Thomas, J. J.; Averbuj, C.; Broo, K. M.; Engelhard, M.; Crowell, E.; Finn, M. G.; Siuzdak, G. Porous Silicon as a Versatile Platform for Laser Desorption/Ionization Mass Spectrometry. Anal. Chem. 2001, 73, 612-619.

28. Enjalbal, C.; Lamaty, F.; Sanchez, P.; Suberchicot, E.; Ribière, P.; Varray, S.; Lazaro, R.; Yadav-Bhatnagar, N.; Martinez J.; Aubagnac, J.-L. Characterization of Soluble Polymer Supported Organic Compounds by LC/Electrospray Ionization MS Towards a Complete Automation of the Liquid-Phase Process in Combinatorial Chemistry. Anal. Chem. 2003, 75, 175-184.

29. Luo, G. H.; Chen, Y.; Siuzdak, G.; Vertes, A. Surface Modification and Laser Pulse Length Effects on Internal Energy Transfer in DIOS. J. Phys. Chem. B 2005, 109, 24450-24456. 